\title{
EL APRENDIZAJE BASADO EN PROYECTOS COMO HERRAMIENTA PARA LA FORMACIÓN EN GANADERÍA DE
} PRECISIÓN

\section{PROJECT-BASED LEARNING AS A TOOL FOR TRAINING ON PRECISION LIVESTOCK FARMING}

Francisco Maroto Molina, Dolores C. Pérez Marín, Ana Garrido Varo, José Emilio Guerrero Ginel, José Antonio Adame Siles y Cecilia Riccioli g02mamof@uco.es

Universidad de Córdoba

Received: 28/06/2017 Accepted: 20/02/2018

\section{Resumen}

Este artículo describe una experiencia en el uso de la metodología docente del aprendizaje basado en proyectos en varias asignaturas de Grado y Máster de la Escuela Técnica Superior de Ingeniería Agronómica y de Montes. Se ha seleccionado el tema de la ganadería de precisión para el desarrollo de los proyectos, en concreto el uso de acelerómetros para la monitorización del movimiento de los animales. Los estudiantes, en grupos de ocho a diez miembros, han diseñado y aplicado un proyecto relativo a las utilidades reales de los datos registrados por collares para vacas que incorporan sensores de posicionamiento y aceleración. Esta metodología ha mejorado la motivación de los estudiantes y algunas de sus habilidades. No obstante, se han encontrado algunos problemas relacionados con la gestión de los grupos de trabajo y con las características de los datos registrados por los collares.

Palabras clave: Innovación docente; Aprendizaje basado en proyectos; Agricultura digital; Tecnología; Ingeniería.

\begin{abstract}
This paper describes an experience in the use of the teaching methodology of project-based learning in several undergraduate and postgraduate courses of the Escuela Técnica Superior de Ingeniería Agronómica y de Montes. Precision Livestock Farming was chosen as the main topic of the projects, specifically the use of accelerometers to monitor animal activity. The students, working in groups of eight to ten members, have designed and applied a project relating real-world utilities for data recorded by GPS + accelerometer cow collars. This methodology has improved motivation and some of students' skills. Nevertheless, some problems have arisen regarding the management of working groups and the characteristics of data recorded by cow collars.
\end{abstract}

Keywords: Teaching and learning innovation; Project-based learning; Digital farming; Technology; Engineering.

\section{INTRODUCCIÓN}

Las directrices marcadas por el Espacio Europeo de Educación Superior (EESS) en lo referente a la necesidad de un cambio profundo en los métodos de enseñanza tradicionales, han supuesto un reto importante para el profesorado universitario, que ha llevado al replanteamiento de sus estrategias didácticas. Afortunadamente, para abordar este cambio, existen métodos de enseñanza-aprendizaje tales como el aprendizaje colaborativo en el aula, el aprendizaje basado en problemas, o el aprendizaje basado en proyectos, al que se refiere el presente artículo.

El aprendizaje basado en proyectos es una metodología docente en la que los estudiantes adquieren un conocimiento profundo de los contenidos impartidos en clase mediante la construcción activa de dicho conocimiento a partir del diseño y aplicación de ideas en proyectos (Krajcik y Blumenfeld, 2006). En el aprendizaje basado en proyectos, el estudiante se enfrenta a un proyecto del mundo real, que es significativo para su formación y similar al que desarrollan los profesionales o científicos del sector. Ello le permite investigar cuestiones, proponer ideas e hipótesis, discutir dichas ideas e ir adaptándolas conforme avanzan en su conocimiento de la temática. Se ha demostrado que los estudiantes de clases que utilizan la metodología del aprendizaje basado en proyectos obtienen mejores calificaciones que los de clases tradicionales (Marx et al. 1997).

El grupo docente 44 de la Universidad de Córdoba lleva años adaptando su metodología docente a los lineamientos del EEES, evolucionando de un modelo basado en las clases magistrales a una combinación de clases interactivas y tareas a realizar fuera del aula. Prueba de ello son los proyectos de innovación docente financiados en las diversas convocatorias del Plan de Innovación Docente de la Universidad de Córdoba: "Introducción progresiva de los denominados resultados de aprendizaje (learningoutcomes) en las enseñanzas universitarias de la UCO. Caso de estudio: resultados del autoaprendizaje en producción animal" (2004/2005); "Redes cooperativas de profesores seniors y juniors para el avance del conocimiento de estrategias innovadoras de auto-aprendizaje en producción animal" (2007/2008); "Estrategias para incrementar la motivación de los estudiantes por el auto-aprendizaje y el aprendizaje permanente" (2009/2010) y "El método del caso como herramienta para la docencia y el aprendizaje en ingeniería de sistemas de producción agroganadera" (2015/2016) (Maroto et al. 2017). En el curso 2016/2017 el grupo docente 44 ha considerado oportuno dar un paso más, complementario a los anteriores, con el empleo de la metodología del aprendizaje basado en proyectos en varias asignaturas 
del Grado de Ingeniería Agroalimentaria y del Medio Rural y del Máster de Ingeniería Agronómica de la Escuela Técnica Superior de Ingeniería Agronómica y de Montes (ETSIAM).

\section{OBjeTIVOS}

Implementar una experiencia de aprendizaje basado en proyectos para mejorar la motivación de los estudiantes de la ETSIAM y su implicación en el proceso de aprendizaje, así como para el desarrollo de sus habilidades en lo referente a la digitalización de los sistemas de producción agroganadera, aspecto de vital importancia en su futuro desarrollo profesional.

\section{METODOLOGÍA}

Se ha utilizado la metodología del aprendizaje basado en proyectos. Esta metodología pedagógica se centra en los estudiantes, que construyen en el conocimiento de forma activa al enfrentarse a problemas o retos del mundo real. Los problemas planteados a los estudiantes pueden ser de diversa índole. De hecho, se pueden encontrar ejemplos diversos de utilización del aprendizaje basado en proyectos en el ámbito de la ingeniería agronómica (de los Ríos et al. 2010; Ortiz, 2014). En el presente proyecto de innovación docente, se ha seleccionado el tema de la ganadería de precisión (precision livestock.farming). Tradicionalmente, la docencia sobre producción animal se ha basado en la explicación de los principios biológicos que rigen la producción ganadera y en la descripción de los sistemas productivos intensivos y extensivos. Entendemos que esta aproximación, si bien resulta básica, puede complementarse con contenidos docentes relacionados con los grandes drivers del futuro de la ganadería. La sostenibilidad económica, medioambiental y social de los sistemas de producción animal, así como su eficacia (cost-effectiveness), en un mundo con una demanda creciente de carne, leche y huevos, son algunos de los grandes retos de la sociedad en general, y de los futuros ingenieros agrónomos en particular. Banzahi et al. (2012) consideran que la ganadería de precisión es un desarrollo prometedor que tiene el potencial de revolucionar la ganadería actual para superar algunos de retos citados. La ganadería de precisión puede definirse como el uso de la tecnología (sensores, TIC, etc) para optimizar la producción de cada animal (Berckmans, 2004). Esta aproximación individual permite mejorar los resultados de las explotaciones en varios aspectos: eficiencia productiva, bienestar animal, reducción de emisiones de efecto invernadero, segmentación del mercado, etc.

Las tecnologías de ganadería de precisión son diversas y abarcan casi todos los ámbitos de la producción animal: alimentación, reproducción, sanidad, etc. Para la ejecución práctica del presente proyecto se ha seleccionado el ámbito de la monitorización de la actividad de los animales mediante el uso de acelerómetros. Se ha pedido a los estudiantes que trabajen en grupos en el diseño y ejecución de un proyecto de ganadería de precisión consistente en el desarrollo de utilidades asociadas los datos de actividad del ganado capturados con acelerómetros de tres ejes. Los estudiantes de la asignatura de Grado han desarrollado el proyecto a un nivel inferior al de los estudiantes de Máster.

\section{DESCRIPCIÓN}

Las actividades llevadas a cabo en el proyecto fueron:

\subsection{INTRODUCCIÓN A LA GANADERÍA DE PRECISIÓN}

Se utilizó una sesión para presentar a los estudiantes el concepto de ganadería de precisión y algunos ejemplos de tecnologías utilizadas actualmente a nivel comercial o que se encuentran en fase de desarrollo. Se pidió a los estudiantes que completaran la información proporcionada en clase a nivel individual. En el caso de los estudiantes de Grado, se les ofreció la opción de desarrollar uno de los trabajos obligatorios de la asignatura en torno a esta temática.

\subsection{VISITA DE CAMPO}


Se organizó una visita a una explotación de vacuno y ovino de carne con objeto de que los estudiantes conocieran el funcionamiento práctico de los sistemas productivos, así como las necesidades y demandas de los ganaderos en relación con las tecnologías de ganadería de precisión.

Se aprovechó esta visita para colocar collares con sensores GPS + acelerómetro (DIGITANIMAL@) a tres vacas de la

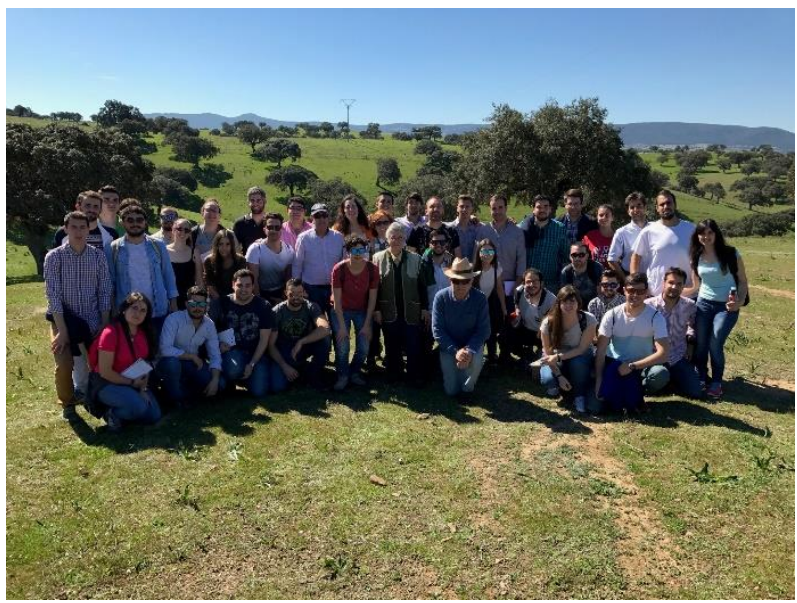

Figura 1. Visita a la explotación El Borde (Ciudad Real).

explotación. Los estudiantes participaron directamente en la colocación de los collares.

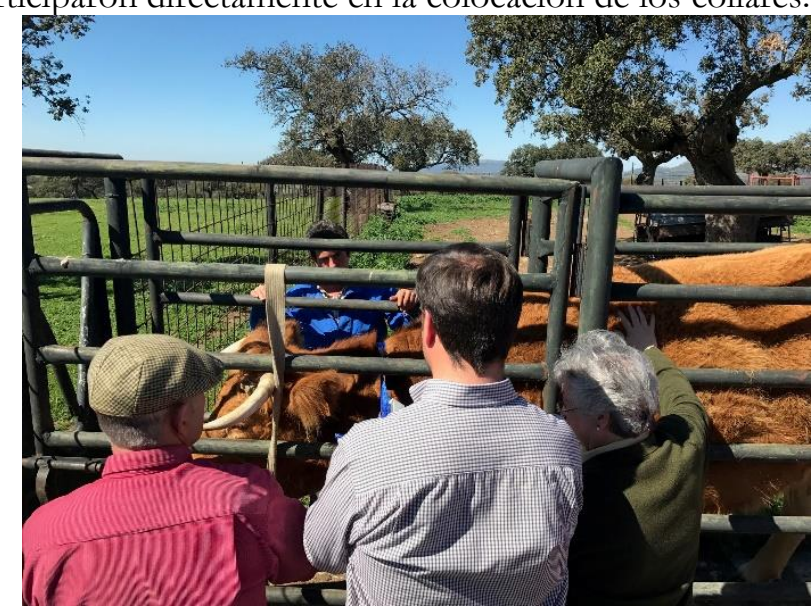

Figura 2. Colocación del collar por un estudiante.

\subsection{INTRODUCCIÓN A LOS ACELERÓMETROS DE TRES EJES}

Se utilizó una sesión de clase para presentar a los estudiantes los fundamentos físicos del funcionamiento de los acelerómetros de tres ejes. Como material docente de apoyo se utilizó la app Accelerometer Analyzer. Los estudiantes instalaron esta aplicación en sus teléfonos móviles y analizaron los cambios en la señal del acelerómetro cuando el teléfono se mueve siguiendo ciertos patrones de movimiento (vertical vs. horizontal, rotación, etc). Además, se realizó un ejercicio consistente en fijar un teléfono móvil a la pierna de un estudiante, quién realizó dos tipos de movimientos: andar y correr. Posteriormente, los datos brutos registrados por el acelerómetro del teléfono durante estos movimientos se entregaron a los estudiantes para que dedujeran el tipo de movimiento a partir del análisis de los datos.

\subsection{DEFINICIÓN DE LOS PROYECTOS}

Se entregaron a los estudiantes las bases de datos generadas por los collares colocados en la visita a la explotación, junto con los metadatos relativos a las características de las vacas sensorizadas (edad, raza, etc) y los sucesos reproductivos (partos, celos, etc) registrados para dichos animales en el periodo de monitorización. Se pidió a los estudiantes que, en grupos de ocho a diez, diseñaran y ejecutaran un proyecto relativo al desarrollo de utilidades asociadas a los datos de aceleración registrados por los collares. Se les proporcionó información sobre las posibles utilidades (detección de celos, caracterización de patrones de pastoreo, etc) y sobre las técnicas estadísticas necesarias para el análisis de los datos.

\subsection{TRABAJO EN LOS PROYECTOS Y TUTORÍAS DE SEGUIMIENTO}

Los estudiantes trabajaron en sus proyectos dentro y fuera del aula. Paralelamente, cada grupo solicitó varias tutorías para comentar con los profesores tanto las ideas de proyectos como las dificultades encontradas durante su desarrollo. 


\subsection{PRESENTACIÓN DE LOS PROYECTOS}

Cada grupo de trabajo presentó oralmente al profesorado y a sus compañeros los proyectos desarrollados. Tras cada presentación tuvo lugar un debate sobre las ventajas e inconvenientes de los proyectos desarrollados por los estudiantes.

\section{RESULTADOS OBTENIDOS}

La experiencia de aprendizaje basado en proyectos llevada a cabo ha mejorado las capacidades de autoaprendizaje de los estudiantes, al mismo tiempo que les ha permitido integrar los conocimientos adquiridos en diversas asignaturas del Grado y/o Máster: producción animal, física, estadística, etc. El trabajo en grupo ha mejorado sus capacidades de organización, si bien el elevado número de integrantes de los grupos de trabajo ha supuesto una dificultad añadida en algunos casos. La metodología del aprendizaje basado en proyectos ha aumentado la motivación de los estudiantes, que han solicitado varias tutorías no obligatorias para avanzar en el desarrollo de sus proyectos. En general, los estudiantes han valorado positivamente la oportunidad de enfrentar problemas reales, así como la visita de campo como herramienta para recabar información de los sistemas de producción ganadera. La necesidad de defender sus proyectos frente a profesores y compañeros ha permitido mejorar las capacidades de comunicación de los estudiantes.

En cuanto a los proyectos desarrollados por los estudiantes, algunos grupos llegaron a desarrollar prototipos de algoritmos para la detección de episodios de alta o baja actividad de los animales, lo cual puede estar relacionado con celos, enfermedades, etc. No obstante, cabe destacar que hubo una pérdida importante de datos procedentes de los collares debido a problemas de cobertura de la red SigFox, utilizada por DIGITANIMAL® para la transmisión inalámbrica de la información. De este modo, aunque la información proporcionada a los estudiantes fue abundante (más de 5000 registros de posición y aceleración) en algunos días la pérdida de registros superó el 50\%, lo cual supuso un reto añadido para el análisis de los datos.

\section{UTILIDAD}

La experiencia descrita en este artículo ha sido útil para los estudiantes desde el punto de vista de la mejora de la motivación por el aprendizaje y el desarrollo de ciertos conocimientos y habilidades. Asimismo, ha sido útil para el grupo docente 44 para seguir avanzando en la mejora de la docencia, tanto a nivel de Grado como de Máster, mediante el uso y validación de nuevas metodologías.

\section{CONCLUSIONES}

El aprendizaje basado en proyectos es una metodología docente muy adecuada para estudiantes de ingeniería agronómica, especialmente para aquellos que tienen una base de conocimiento razonable y están cercanos a enfrentar problemas reales en su desarrollo profesional, como es el caso de los estudiantes de Máster. La metodología mejora la motivación de los estudiantes y su implicación en el proceso de aprendizaje, así como algunas de sus habilidades (manejo de hardware y software, análisis de datos, etc). No obstante, cuando se implementa en base al trabajo en grupo, pueden surgir dificultades relacionadas con la disparidad de propuestas respecto al proyecto a desarrollar y al reparto de tareas entre los miembros de los grupos. Por ello, es recomendable que la implementación de esta metodología vaya acompañada de una formación en gestión de grupos de trabajo.

\section{AGRADECIMIENTOS}

Los autores agradecen a la Universidad de Córdoba la financiación recibida para la ejecución del proyecto de innovación docente titulado "Aprendizaje basado en proyectos como herramienta para la formación en ganadería de precisión" (2016-1-5006) durante el curso 2016/2017.

\section{BIBLIOGRAFÍA}

BAnZAhi, T.M., Lehr, H., Black, J.L., CrabTree, H., SCHOFIEld, P., TsCHARKe, M., Berckmans, D. "Precision Livestock Farming: an international review on scientific and commercial aspects", International Journal of Agricultural and Biological Engineering 2012/5 (3), p. 1-9.

BERCKMANS, D. “Automated on-line monitoring of animals by Precision Livestock Farming”, in F. MADEC (ed.), International Society for Animal Hygiene, Saint Malo, 2004, p. 27-30.

DE los Ríos, I., CAZORLA, A., DíAz Puente, J.M., YagüE, J.L. "Project-based learning in engineering higher education: two decades of teaching competences in real environments", Procedia Social and Behavioral Sciences 2010/2 (2), p. 1368-1378.

KrajCiK, J.S., Blumenfeld, P.C. "Project-based learning (Chapter 19)", The Cambridge Handbook of the Learning Sciences, Cambridge, 2006, p. 317-334. 
El aprendizaje basado en proyectos como herramienta para la formación...

MAROTO, F., GÓMEZ, A., GARRIDO, A., PÉREZ, D.C., DE PEDRO, E. “El método del caso como herramienta para la docencia y el aprendizaje en ingeniería de sistemas de producción ganadera", Revista de Innovación y Buenas Prácticas Docentes 2017/1, p. 4550.

MarX, R.W., Blumenfeld, P.C., KRAJCIK, J.S., SOlOWAY, E. "Enacting project-based science", Elementary School Journal 1997/97 (4), p. 341-358.

ORTIZ, L. Incorporación de la perspectiva emprendedora en un centro de educación superior de Ingeniería Agronómica e Ingeniería de Montes. Tesis Doctoral, Córdoba, 2014. 